\title{
Deciding first-order properties of locally tree-decomposable structures
}

\author{
Markus Frick Martin Grohe \\ Institut für Mathematische Logik, Eckerstr. 1, 79104 Freiburg, Germany \\ Email: \{frick,grohe\}@logik.mathematik.uni-freiburg.de
}

October 30, 2018

\begin{abstract}
We introduce the concept of a class of graphs, or more generally, relational structures, being locally tree-decomposable. There are numerous examples of locally tree-decomposable classes, among them the class of planar graphs and all classes of bounded valence or of bounded tree-width. We also consider a slightly more general concept of a class of structures having bounded local tree-width.

We show that for each property $\varphi$ of structures that is definable in first-order logic and for each locally tree-decomposable class $\mathrm{C}$ of graphs, there is a linear time algorithm deciding whether a given structure $\mathcal{A} \in \mathrm{C}$ has property $\varphi$. For classes $\mathrm{C}$ of bounded local tree-width, we show that for every $k \geq 1$ there is an algorithm that solves the same problem in time $O\left(n^{1+(1 / k)}\right)$ (where $n$ is the cardinality of the input structure).
\end{abstract}

\section{Introduction}

It is an important task in the theory of algorithms to find feasible instances of otherwise intractable algorithmic problems. A notion that has turned out to be extremely useful in this context is that of tree-width of a graph. 3-COLORABILITY, HAMILTONICITY, and many other NP-complete properties of graphs can be decided in linear time when restricted to graphs whose tree-width is bounded by a fixed constant (see [7] for a survey).

Courcelle [8] proved a meta-theorem, which easily implies numerous results of the abovementioned type: Let $w \geq 1$ and $\varphi$ be a property of graphs that is definable in monadic second-order logic. Then $\varphi$ can be decided in linear time on graphs of tree-width at most $w$. As a matter of fact, this result does not only hold for graphs, but for arbitrary relational structures. Although Courcelle's theorem does not give practical algorithms, because the hidden constants are too big, it is still useful since it gives a simple way to recognize a property as being linear time decidable on graphs of bounded tree-width. Once this has been done, a more refined analysis using the combinatorics of the particular property may yield a practical algorithm.

Though maybe the most successful, bounded tree-width is not the only restriction on graphs that makes algorithmic tasks easier. Other useful restrictions are planarity or bounded valence. For example, consider the problem $k$-Dominating SET for a fixed $k$. (Given a graph $\mathcal{G}$, is there a set $D$ of at most $k$ vertices of $\mathcal{G}$ such that every vertex of $\mathcal{G}$ is either equal or adjacent to a vertex in $D$ ?) To solve $k$-Dominating SET in general, we do not know much better than just trying all $O\left(n^{k}\right)$ candidate sets ( $n$ always denotes the number of vertices of the input graph). However, on planar graphs $k$-DOMINATING SET can be solved in time $O\left(11^{k} n\right)$, and on graphs of valence at most $l$, it can be solved in time $O\left((l+1)^{k} n\right)[10]$.

Unfortunately, the analogue of Courcelle's theorem does not hold for planar graphs or classes of bounded valence; 3-COLORABILITY is a monadic second-order definable property that remains NP-complete when restricted to the class of planar graphs of valence at most 4 [18]. Instead of monadic second-order, we study the complexity of first-order definable properties.

A preliminary version appeared in Proceedings of the 26th International Colloquium on Automata, Languages, and Programming, Lecture Notes in Computer Science 1644, pp.331-340. (c) Springer-Verlag 1999 
Seese was the first to give a meta-theorem in the style of Courcelle's theorem for a more general class of structures; in [25] he proved that for every $l \geq 1$ and for every first-order definable property of structures there is a linear time algorithm that decides whether a given structure of valence at most $l$ has this property.

An observation that has been used for various algorithms on planar graphs (essentially it goes back to Baker (5]) is that there is a bound on the tree-width of a planar graph only depending on its diameter. A different way to see this is that a local neighborhood of a vertex in a planar graph has tree-width bounded by a number only depending on the radius of this neighborhood. As a matter of fact, given a planar graph $\mathcal{G}$ we can compute in linear time a family of subgraphs of bounded tree-width such that a suitably big neighborhood of every vertex of $\mathcal{G}$ is completely contained in one of these subgraphs.

We call classes of graphs admitting such a covering algorithm locally tree-decomposable (a precise definition is given in Section 6. Examples of locally tree-decomposable classes of graphs are all classes of bounded genus, bounded valence, and bounded tree-width. The concept easily generalizes to arbitrary relational structures.

Eppstein [12] considered a closely related, though slightly weaker concept he called the diametertreewidth property (we call this property bounded local tree-width and refer the reader to Section 5 for the definition). Eppstein proved that the subgraph isomorphism problem for a fixed subgraph $\mathcal{H}$, asking whether a given graph $\mathcal{G}$ contains $\mathcal{H}$, is solvable in linear time when restricted to graphs $\mathcal{G}$ contained in a class of graphs that is closed under taking minors and has bounded local tree-width. It is not hard to see that every class $\mathrm{C}$ of graphs that is closed under taking minors and has bounded local tree-width is locally tree-decomposable (cf. Lemma 6.6).

Thus our main result goes much further:

Theorem 1.1. Let $\mathrm{C}$ be a class of relational structures that is locally tree-decomposable and $\varphi$ a property definable in first-order logic. Then there is a linear time algorithm deciding whether a given structure $\mathcal{A} \in \mathrm{C}$ has property $\varphi$.

It may be worth mentioning that in the terminology of [27, our result can be rephrased as follows: When restricted to a locally tree-decomposable class of structures, the data complexity of first-order logic is in linear time.

Examples of first-order definable properties are $k$-DOMINATING-SET and $k$-INDEPENDENT-SET for a fixed $k, \mathcal{H}$-SUbGRAPH-ISOMORPHISM (Given $\mathcal{G}$, is $\mathcal{H} \subseteq \mathcal{G}$ ?) and $\mathcal{H}$-HomOMORPHISM (Given $\mathcal{G}$, is there a homomorphism $h: \mathcal{H} \rightarrow \mathcal{G}$ ?) for a fixed $H,(\mathcal{H}, \mathcal{K}$ )-EXTENSION (Given $\mathcal{G}$, is every $\mathcal{H} \subseteq \mathcal{G}$ contained in some $\mathcal{K} \subseteq \mathcal{G}$ ?) for fixed $\mathcal{H} \subseteq \mathcal{K}$. Let us also give a few examples of a problems defined on other relational structures than graphs. For $k \geq 1, k$-SET-COVER is the problem of deciding whether a given family $\mathcal{F}$ of sets has a subfamily $\mathcal{S}$ of size at most $k$ such that $\bigcup \mathcal{S}=\bigcup \mathcal{F}$. For $d \geq 1,(k, d)$-CirCUIT-S ATISFIABILITY is the problem of deciding whether a given Boolean circuit of depth at most $d$ has a satisfying assignment in which at most $k$ input gates are set to 'true'. Both $k$-SET-Cover and $(k, d)$-CirCUIT-SATISFIABILITY can be seen as first-order definable problems on certain relational structures. Thus our theorem implies, for example, that $k$-SET-COVER can be solved in linear time for set systems where each element is only contained in a bounded number of sets, and that $(k, d)$-CIRCUIT-SATISFIABILITY can be solved in linear time for circuits whose underlying graph is planar. Of course problems like SUBGRAPH-ISOMORPHISM, HOMOMORPHISM, EXTENSION can be generalized arbitrary relational structures.

As a last example, let us consider the problem of evaluating a (Boolean) database query formulated in the relational calculus against a relational database. Since relational calculus is the same as first-order logic, and relational databases are just finite relational structures, our theorem applies and shows, for example, that Boolean relational calculus queries can be evaluated in linear time on databases whose underlying graph is planar. As a matter of fact, this last example was one of our main motivation for starting this research. It seems that when storing geographical data such as road maps, planar structures come up quite naturally.

Thus our theorem gives a unifying framework for various results solving concrete problems on specific locally tree-decomposable classes such as the class of planar graphs. In addition, it yields a number of new results of this type.

Using the same techniques, we prove another theorem that applies to the even more general context of classes of structures of bounded local tree-width: 
Theorem 1.2. Let $\mathrm{C}$ be a class of relational structures of bounded local tree-width and $\varphi$ a first-order definable property. Then for every $k \geq 1$ there is an algorithm deciding whether a given structure $\mathcal{A} \in \mathrm{C}$ has property $\varphi$ in time $O\left(n^{1+(1 / k)}\right)$.

The complexity of first-order properties of relational structures has been studied under various aspects. It is long known that every first-order property of graphs can be decided in polynomial time, actually in $\mathrm{AC}_{0}$ [ 2 , 19]. A question closer to our theorem is whether deciding first-order properties is fixed-parameter tractable, that is, whether there is a fixed $c$ such that every first-order property of finite relational structures can be decided in time $O\left(n^{c}\right)$. This question has been brought up by Yannakakis [28]. The theory of fixed-parameter tractability gives some evidence that the answer is no, as has been independently proved by Downey, Fellows, Taylor [11] and Papadimitriou, Yannakakis [20] (deciding first-order properties is AW[1]-complete). Theorem 1.2 shows that deciding first-order properties of structures in a class of bounded local tree-width is fixed-parameter tractable. Furthermore, it has been used in [16] to show that for every class $\mathrm{C}$ of graphs such that there is some graph that is not a minor of any graph in $\mathrm{C}$, deciding first-order properties of graphs in $\mathrm{C}$ is fixed-parameter tractable.

The proofs of our results combine three main ingredients: a refinement of Courcelle's Theorem [8] mentioned above, Gaifman's Theorem [17] stating that first-order properties are local, and algorithmic techniques based on ideas of Baker [5] and Eppstein [12]. To prove Theorem 1.2, we also use covering techniques due to Awerbuch and Peleg [4, 21].

\section{Preliminaries}

A vocabulary is a finite set of relation symbols. Associated with every relation symbol $R$ is a positive integer called the arity of $R$. In the following, $E$ always denotes a binary relation symbol and $\tau$ a vocabulary.

A $\tau$-structure $\mathcal{A}$ consists of a non-empty set $A$, called the universe of $\mathcal{A}$, and a relation $R^{\mathcal{A}} \subseteq A^{r}$ for each $r$-ary relation symbol $R \in \tau$. If $\mathcal{A}$ is a $\tau$-structure and $B \subseteq A$, then $\langle B\rangle^{\mathcal{A}}$ denotes the substructure induced by $\mathcal{A}$ on $B$, that is, the $\tau$-structure $\mathcal{B}$ with universe $B$ and $R^{\mathcal{B}}:=R^{\mathcal{A}} \cap B^{r}$ for every $r$-ary $R \in \tau$.

For instance, we consider graphs as $\{E\}$-structures $\mathcal{G}=\left(G, E^{\mathcal{G}}\right)$, where the binary relation $E^{\mathcal{G}}$ is symmetric and anti-reflexive (i.e. graphs are undirected and loop-free). As another example, we can view hypergraphs as $\{E, P\}$-structures, where $E$ is binary and $P$ unary. A hypergraph with vertices $V$ and hyperedges $\mathcal{H} \subseteq \operatorname{Pow}(V)$ is modeled by the $\{E, P\}$-structure $(V \cup \mathcal{H},\{(v, H) \mid v \in H\}, V)$.

In this paper we only consider finite structures. Let us remark that all the results of this paper remain true if we also admit constants in our structures. We restrict our attention to the relational case because constants would not give us additional insights.

The formulas of first-order logic FO are build up in the usual way from an infinite supply of variables denoted by $x, y, x_{1}, \ldots$, the equality symbol $=$ and relation symbols of a vocabulary $\tau$, the connectives $\wedge, \vee, \neg, \rightarrow$, and the quantifiers $\forall, \exists$ ranging over the universe of the structure. For example, the first-order sentence

$$
\varphi:=\forall x_{1} \forall x_{2} \forall x_{3}\left(\left(E x_{1} x_{2} \wedge E x_{1} x_{3} \wedge E x_{2} x_{3}\right) \rightarrow \exists y\left(E x_{1} y \wedge E x_{2} y \wedge E x_{3} y\right)\right)
$$

says that every triangle of a graph is contained in a $K_{4}$ (a complete graph on four vertices). The formula

$$
P x \wedge \neg \exists y \exists z(\neg y=z \wedge E x y \wedge E x z)
$$

defines the set of all vertices $x$ of a hypergraph that are contained in at most one hyperedge.

A free variable in a first-order formula is a variable $x$ not in the scope of a quantifier $\exists x$ or $\forall x$. A sentence is a formula without free variables. The notation $\varphi\left(x_{1}, \ldots, x_{k}\right)$ indicates that all free variables of the formula $\varphi$ are among $x_{1}, \ldots, x_{k}$; it does not necessarily mean that the variables $x_{1}, \ldots, x_{k}$ all appear in $\varphi$. For a formula $\varphi\left(x_{1}, \ldots, x_{k}\right)$, a structure $\mathcal{A}$, and $a_{1}, \ldots, a_{k} \in A$ we write $\mathcal{A} \models \varphi\left(a_{1}, \ldots, a_{k}\right)$ to say that $\mathcal{A}$ satisfies $\varphi$ if the variables $x_{1}, \ldots, x_{k}$ are interpreted by the vertices $a_{1}, \ldots, a_{k}$, respectively.

Example 2.1. In this example we show how to model the $k$-SET-COVER problem mentioned in the introduction by a first-order definable problem. We can view a family $\mathcal{F}$ of a sets as the hypergraph whith vertex set $\bigcup \mathcal{F}$ and edge set $\mathcal{F}$. 
Let

$$
\varphi_{k}:=\exists x_{1} \ldots x_{k} \forall y\left(P y \rightarrow\left(E y x_{1} \vee \ldots \vee E y x_{k}\right)\right) .
$$

Then the hypergraph corresponding to the family $\mathcal{F}$ satisfies $\varphi_{k}$ if and only if there exists an $\mathcal{S} \subseteq \mathcal{F}$ of cardinality $|\mathcal{S}|=k$ such that $\bigcup \mathcal{S}=\bigcup \mathcal{F}$.

We often denote tuples $\left(a_{1}, \ldots, a_{k}\right)$ of elements of a set $A$ by $\bar{a}$, and we write $\bar{a} \in A$ instead of $\bar{a} \in A^{k}$. Similarly, we denote tuples of variables by $\bar{x}$.

Our underlying model of computation is the standard RAM-model with addition and subtraction as arithmetic operations (cf. [1, 26]). In our complexity analysis we use the uniform cost measure. Structures are represented on a RAM in a straightforward way by listing all elements of the universe and then all tuples in the relations. For details we refer the reader to [15]. We define the size of a $\tau$-structure $\mathcal{A}$ to be $\|\mathcal{A}\|:=|A|+\sum_{R \in \tau \text {-ary }} r \cdot\left|R^{\mathcal{A}}\right|$; this is the length of a reasonable representation of $\mathcal{A}$ (if we suppress details that are inessential for us).

\section{Gaifman's Theorem}

The Gaifman graph of a $\tau$-structure $\mathcal{A}$ is the graph $\mathcal{G}_{\mathcal{A}}$ with vertex set $G_{\mathcal{A}}:=A$ and an edge between two vertices $a, b \in A$ if there exists an $R \in \tau$ and a tuple $\left(a_{1}, \ldots, a_{k}\right) \in R^{\mathcal{A}}$ such that $a, b \in\left\{a_{1}, \ldots, a_{k}\right\}$. The distance $d^{\mathcal{A}}(a, b)$ between two elements $a, b \in A$ of a structure $\mathcal{A}$ is the length of the shortest path in $\mathcal{G}_{\mathcal{A}}$ connecting $a$ and $b$. For $r \geq 1$ and $a \in A$ we define the $r$-neighborhood of $a$ in $\mathcal{A}$ to be $N_{r}^{\mathcal{A}}(a):=$ $\left\{b \in A \mid d^{\mathcal{A}}(a, b) \leq r\right\}$. For a subset $B \subseteq A$ we let $N_{r}^{\mathcal{A}}(B):=\bigcup_{b \in B} N_{r}^{\mathcal{A}}(b)$.

For every $r \geq 0$ there is a first-order formula $\delta_{r}(x, y)$ such that for all $\tau$-structures $\mathcal{A}$ and $a, b \in A$ we have $\mathcal{A} \models \delta_{r}(a, b) \Longleftrightarrow d^{\mathcal{A}}(a, b) \leq r$. For example, if $\tau=\{E, T\}$ consists of a binary and a ternary relation symbol, we let

$$
\begin{aligned}
& \delta_{0}(x, y):=(x=y) \\
& \delta_{1}(x, y):=\delta_{0}(x, y) \vee E x y \vee E y x \vee \exists z(T x y z \vee T y x z \vee T x z y \vee T y z x \vee T z x y \vee T z y x) \\
& \delta_{2}(x, y):=\delta_{0}(x, y) \vee \delta_{1}(x, y) \vee \exists z\left(\delta_{1}(x, z) \wedge \delta_{1}(z, y)\right)
\end{aligned}
$$

In the following, we write $d(x, y) \leq r$ instead of $\delta_{r}(x, y)$ and $d(x, y)>r$ instead of $\neg \delta_{r}(x, y)$.

If $\varphi(x)$ is a first-order formula, then $\varphi^{N_{r}(x)}(x)$ is the formula obtained from $\varphi(x)$ by relativizing all quantifiers to $N_{r}(x)$, that is, by replacing every subformula of the form $\exists y \psi(x, y, \bar{z})$ by $\exists y(d(x, y) \leq$ $r \wedge \psi(x, y, \bar{z}))$ and every subformula of the form $\forall y \psi(x, y, \bar{z})$ by $\forall y(d(x, y) \leq r \rightarrow \psi(x, y, \bar{z}))$. A formula $\psi(x)$ of the form $\varphi^{N_{r}(x)}(x)$, for some $\varphi(x)$, is called $r$-local. The basic property of $r$-local formulas $\psi(x)$ is that it only depends on the $r$-neighborhood of $x$ whether they hold at $x$ or not, that is, for all structures $\mathcal{A}$ and $a \in A$ we have $\mathcal{A} \models \psi(a) \Longleftrightarrow\left\langle N_{r}^{\mathcal{A}}(a)\right\rangle \models \psi(a)$.

Theorem 3.1 (Gaifman [17]). Every first-order sentence is equivalent to a Boolean combination of sentences of the form

$$
\exists x_{1} \ldots \exists x_{k}\left(\bigwedge_{1 \leq i<j \leq k} d\left(x_{i}, x_{j}\right)>2 r \wedge \bigwedge_{1 \leq i \leq k} \psi\left(x_{i}\right)\right),
$$

for suitable $r, k \geq 1$ and an $r$-local $\psi(x)$.

\section{Tree-width}

A tree is an acyclic graph. A tree-decomposition of a $\tau$-structure $\mathcal{A}$ is a pair $\left(\mathcal{T},\left(B_{t}\right)_{t \in T}\right)$, where $\mathcal{T}$ is a tree and $\left(B_{t}\right)_{t \in T}$ a family of subsets of $A$ (called the blocks of the decomposition) such that

(1) For every $a \in A$, the set $\left\{t \in T \mid a \in B_{t}\right\}$ is non-empty and connected in $\mathcal{T}$ (that is, induces a subtree). 
(2) For every $R \in \tau$ and all $\bar{a} \in R^{\mathcal{A}}$ there is a $t \in T$ such that $\bar{a} \in B_{t}$.

The width of a tree-decomposition $\left(\mathcal{T},\left(B_{t}\right)_{t \in T}\right)$ is $\max \left\{\left|B_{t}\right| \mid t \in T\right\}-1$. The tree-width $\operatorname{tw}(\mathcal{A})$ of $\mathcal{A}$ is the minimal width of a tree-decomposition of $\mathcal{A}$.

We occasionally use the following simple fact (cf. [24]).

Lemma 4.1. Let $w \geq 1$ and $\tau$ a vocabulary. Then there is a constant $c$ such that for every $\tau$-structure $\mathcal{A}$ of tree-width at most $w$ we have $\|\mathcal{A}\| \leq c|A|$.

Bodlaender [6] proved that for each $w \geq 1$ there is a linear time algorithm that, given a graph $\mathcal{G}$, either computes a tree-decomposition of $\mathcal{G}$ of width at most $w$, or rejects $\mathcal{G}$ if $\operatorname{tw}(\mathcal{G})>w$. This result is underlying most of the linear time algorithms on graphs of bounded tree-width. Using the well-known fact that a structure $\mathcal{A}$ has the same tree-width as its Gaifman graph $\mathcal{G}_{\mathcal{A}}$, Bodlaender's result can easily be extended to arbitrary relational structures.

Recall Courcelle's theorem that we mentioned in the introduction:

Theorem 4.2 (Courcelle [8]). Let $w \geq 1$. Then for every sentence $\varphi$ of monadic second-order logic there is a linear time algorithm that decides whether a given structure $\mathcal{A}$ of tree-width at most $w$ satisfies $\varphi$.

Monadic second-order logic is an extension of first-order logic that also allows quantification over sets.

Using known techniques for algorithms on graphs of bounded tree-width, it is not hard to prove the following lemma (see [15]). We are only going to use the first-order version of the lemma later.

Lemma 4.3. Let $w \geq 1$. Then for every formula $\varphi(x)$ of monadic second-order logic there is a linear time algorithm that, given a graph $G$ of tree-width at most $w$, computes the $\operatorname{set} \varphi(\mathcal{A}):=\left\{a \in V^{G} \mid G \models \varphi(a)\right\}$.

\section{Local Tree-Width}

Definition 5.1. (1) The local tree-width of a structure $\mathcal{A}$ is the function $1 \mathrm{ltw}^{\mathcal{A}}: \mathbb{N} \rightarrow \mathbb{N}$ defined by

$$
\operatorname{ltw}^{\mathcal{A}}(r):=\max \left\{\operatorname{tw}\left(\left\langle N_{r}^{\mathcal{A}}(a)\right\rangle\right) \mid a \in A\right\} .
$$

(2) A class $\mathrm{C}$ of structures has bounded local tree-width if there is a function $f: \mathbb{N} \rightarrow \mathbb{N}$ such that $\operatorname{ltw}^{\mathcal{A}}(r) \leq f(r)$ for all $\mathcal{A} \in \mathrm{C}, r \in \mathbb{N}$.

Example 5.2. Structures of bounded tree-width. Let $\mathcal{A}$ be a structure of tree-width at most $k$. Then $\operatorname{ltw}^{\mathcal{A}}(r) \leq k$ for all $r \in \mathbb{N}$.

The valence of a structure $\mathcal{A}$ is the maximal number of neighbors of a vertex $a \in A$ in the Gaifman graph $\mathcal{G}_{\mathcal{A}}$, i.e. $\max _{a \in A}\left|\left\{b \mid(a, b) \in E^{\mathcal{G}_{\mathcal{A}}}\right\}\right|$.

Example 5.3. Structures of bounded valence. Let $\mathcal{A}$ be a structure of valence at most $l$, for an $l \geq 1$. Then $\operatorname{ltw}^{\mathcal{A}}(r) \leq l(l-1)^{r-1}$ for all $r \in \mathbb{N}$.

Example 5.4 (Robertson and Seymour [23]). Planar Graphs. The class of planar graphs has bounded local tree-width. More precisely, for every planar graph $G$ and $r \geq 1$ we have $\operatorname{ltw}^{G}(r) \leq 3 r$.

Example 5.5 (Eppstein [12]). Graphs of bounded genus. Let $S$ be a surface. Then the class of all graphs embeddable in $S$ has bounded local tree-width. More precisely, there is a constant $c$ such that for all graphs $G$ embeddable in $S$ and for all $r \geq 0$ we have $\operatorname{ltw}^{G}(r) \leq c \cdot g(S) \cdot r$.

Example 5.6. We can view a simplicial complex as a hypergraph whose vertices are the corners of the complex. Then it is easy to see that the class of all simplicial complexes homeomorphic to a 2-manifold has bounded local tree-width. 
Recall that a minor of a graph $\mathcal{G}$ is a graph $\mathcal{H}$ that is obtained from a subgraph of $\mathcal{G}$ by contracting edges. The class of planar graphs, and, more generally, the classes of graphs of bounded genus are examples of classes of graphs that are closed under taking minors. Eppstein gave the following nice characterization of all classes of graphs of bounded loocal tree-width that are closed under taking minors. An apex graph is a graph $G$ that has a vertex $v \in V^{G}$ such that $G \backslash\{v\}$ is planar.

Theorem 5.7 (Eppstein [13, 12]). Let $\mathrm{C}$ be a minor-closed class of graphs. Then $\mathrm{C}$ has bounded local tree-width if, and only if, $\mathrm{C}$ does not contain all apex graphs.

This yields further examples of classes of graphs of bounded local tree-width. For example, for every $n \geq 1$, the class of all graphs that do not contain the graph $K_{3, n}$ as a minor has bounded local tree-width. ( $K_{m, n}$ denotes the complete bipartite graph with parts of size $m$ and $n$, respectively.)

Note that a structure has the same local tree-width as its Gaifman graph, so Examples 5.4 and 5.5 and Theorem 5.7 also give rise to examples of classes of structures of arbitrary vocabularies that have bounded local tree-width.

One of the nice things about bounded local tree-width is that the notion is quite flexible. Think of a structure modeling a subway map. The Gaifman graph of this structure will probably be close to planar, but there may be some edges crossing. Therefore, it may be the case that planar graph algorithms do not apply, although the graph is almost planar. On the other hand, the local tree-width of the graph is probably very close to that of a planar graph, and we can still use our algorithms for graphs of bounded local tree-width.

\section{Neighborhood and tree covers}

To explore the local tree-likeness of structures of bounded local tree-width we need to cover them by structures of small tree-width in a suitable way. The most general approach is to use sparse neighborhood covers, as they have been studied, for instance, in [4, 3, 21].

Definition 6.1. Let $r, s \geq 0$. An $(r, s)$-neighborhood cover of a structure $\mathcal{A}$ is a family $\mathcal{N}$ of subsets of $A$ with the following properties:

(1) For every $a \in A$ there exists a $N \in \mathcal{N}$ such that $N_{r}^{\mathcal{A}}(a) \subseteq N$.

(2) For every $N \in \mathcal{N}$ there exists an $a \in A$ such that $N \subseteq N_{s}^{\mathcal{A}}(a)$.

We define the size of a family $\mathcal{N}$ of sets to be $\|\mathcal{N}\|:=\sum_{N \in \mathcal{N}}|N|$. Recall that the size of a $\tau$ structure $\mathcal{A}$ is $\| \mathcal{A}||=|A|+\sum_{R \in \tau \text { r-ary }} r\left|R^{\mathcal{A}}\right|$. The algorithm of the following lemma is an adaptation of an algorithm due to Peleg [21] to our situation. We think it is worthwhile to present our version of the algorithm in some detail.

Lemma 6.2 (Peleg [21]). Let $k \geq 1$. Then there is an algorithm that, given a graph $\mathcal{G}$ and an $r \geq 1$, computes an $(r, 2 k r)$-neighborhood cover $\mathcal{N}$ of $\mathcal{G}$ of size $\|\mathcal{N}\|=O\left(|G|^{1+(1 / k)}\right)$ in time $O\left(\sum_{N \in \mathcal{N}}\left\|\langle N\rangle^{\mathcal{G}}\right\|\right)$.

Proof: The algorithm is described in Figure 11. It iteratively computes a neighborhood cover $\mathcal{N}$, maintaining a set $H$ of vertices whose $r$-neighborhood has not yet been covered by a set in $\mathcal{N}$. In each iteration step of the main loop in Lines 3-13, the algorithm picks an arbitrary vertex $a \in H$ and starts to compute increasing neighborhoods of $a$ (in Lines 6-10) until a certain threshold is reached (cf. Line 10). Then it adds the computed set $N$ to the cover $\mathcal{N}$ and removes all points whose neighborhood has now been covered from $H$, before it goes to the next iteration of the main loop. This process is repeated until $H$ is empty.

Now let $\mathcal{G}$ be a graph, $n:=|G|$, and $r \geq 1$. Let $\mathcal{N}$ be the cover computed by the algorithm.

Claim 1. For every $a \in G$ there exists a $N \in \mathcal{N}$ such that $N_{r}(a) \subseteq N$.

Proof: An element $a$ is removed from the set $H$ of uncovered elements in Line 12 if it belongs to a set $L$ such that $N=N_{r}^{\mathcal{G}}(L)$ has been added to $\mathcal{N}$. Of course this $N$ contains $N_{r}^{\mathcal{G}}(a)$. This proves Claim 1.

Claim 2. For every $N \in \mathcal{N}$ there exists an $a \in G$ such that $N \subseteq N_{2 k r}^{\mathcal{G}}(a)$. 


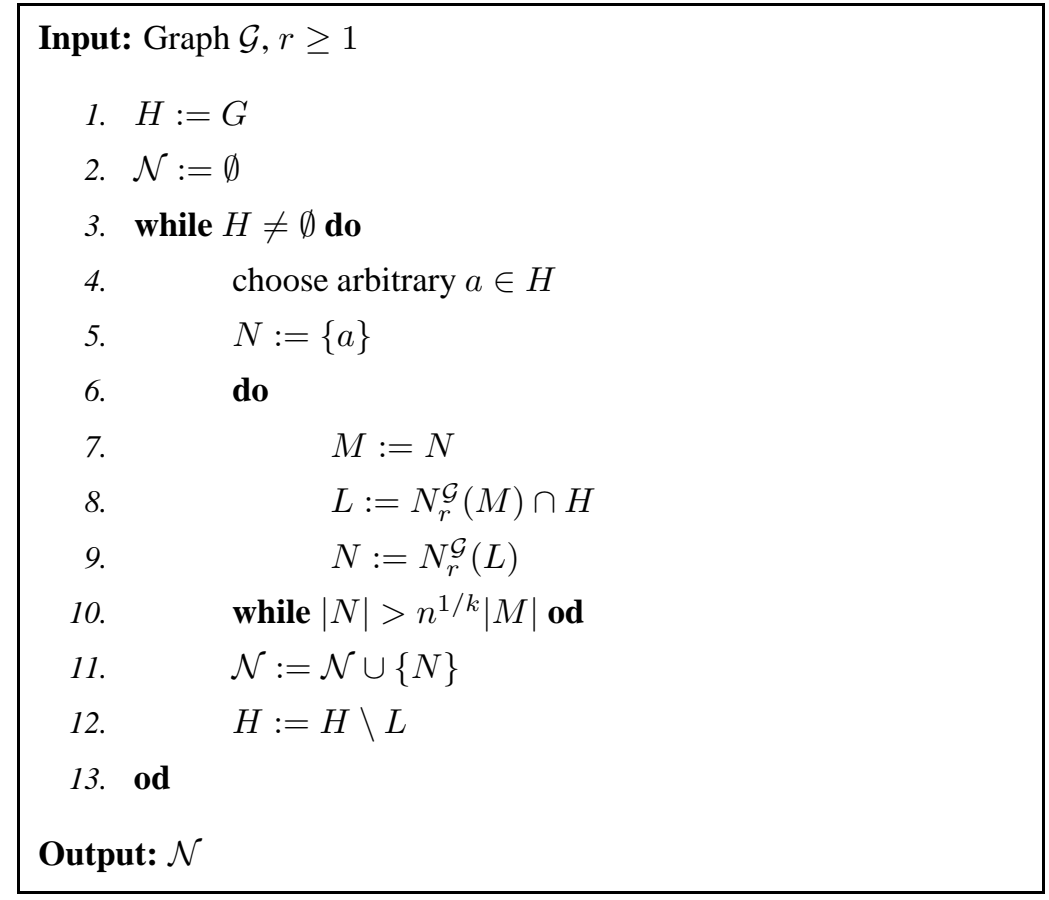

Figure 1.

Proof: We consider the iteration of the main loop that leads to the definition of $N$. Let $a$ be the element chosen in Line 4, and let $N_{0}:=\{a\}$. Let $l \geq 1$ be the number of times the loop in Lines 6-10 is repeated. For $1 \leq i \leq l$, let $N_{i}$ be the value of $N$ after the $i$ th iteration. Then for $1 \leq i \leq l-1$ we have $\left|N_{i}\right|>n^{1 / k}\left|N_{i-1}\right|$, and therefore $\left|N_{i}\right|>n^{i / k}$. Thus $l \leq k$.

Furthermore, it is easy to see that for $1 \leq i \leq l$ we have $N_{i} \subseteq N_{2 i r}^{\mathcal{G}}(a)$. This implies Claim 2.

Claims 1 and 2 show that $\mathcal{N}$ is indeed an $(r, 2 k r)$-neighborhood cover of $\mathcal{G}$. The following Claim 3 shows that the cover is not too large.

Claim 3. $\|\mathcal{N}\| \leq n^{1+(1 / k)}$.

Proof: For $N \in \mathcal{N}$, and let $M$ be the corresponding set that is computed in the last iteration of the loop in Lines 6-10 that let to $N$ (i.e. $M$ is the value of $N$ after the second but last iteration of the loop).

We first show that for distinct $N_{1}, N_{2} \in \mathcal{N}$ we have $M_{1} \cap M_{2}=\emptyset$. To see this, suppose that $N_{1}$ is computed first. Let $H_{1}$ be the value of $H$ after the iteration of the main loop in which $N_{1}$ has been computed. Note that for every $a \in M_{1}$ and $b \in H_{1}$ we have $d^{\mathcal{G}}(a, b)>r$. Moreover, $M_{2} \subseteq N_{2} \subseteq$ $N_{r}^{\mathcal{G}}\left(H_{1}\right)$. Thus $M_{1} \cap M_{2}=\emptyset$.

Noting that by the condition of Line 10 , for all $N \in \mathcal{N}$ we have $|N| \leq n^{1 / k}|M|$, we obtain

$$
\|\mathcal{N}\|=\sum_{N \in \mathcal{N}}|N| \leq n^{1 / k} \sum_{N \in \mathcal{N}}|M| \leq n^{1 / k} \cdot n
$$

The last inequality holds because the $M$ are disjoint subsets of $G$. This proves Claim 3 .

It remains to estimate the running time of the algorithm. We claim that each iteration of the main loop requires time $O\left(\langle N\rangle^{\mathcal{G}}\right)$, for the $N$ added to $\mathcal{N}$ in this iteration. To see this, note that essentially we have to do a breadth-first search on $N$ starting in $a$. To compute $L$ in Line 8, we may have to explore some edges not contained in $\langle L\rangle$. However, all these edges belong to $\left\langle N_{r}^{\mathcal{G}}(L)\right\rangle=\langle N\rangle$.

It may seem that to check the condition of Line 10 we need multiplication, which is not available as basic operation of a standard RAM. However, before we start the main computation we can produce tables 
that store the values $m^{l}$ and $m^{l} \cdot n$ for $1 \leq l \leq k, 1 \leq m \leq n$ in linear time on a standard RAM. (We use the fact that

$$
(m+1)^{l}=\sum_{\left(\epsilon_{1}, \ldots, \epsilon_{l}\right) \in\{0,1\}^{l}} m^{\sum_{i=1}^{l} \epsilon_{i}}
$$

to inductively compute the tables. Remember that we treat $k$ as a constant.) Then we can use these tables to check the condition of Line 10 in constant time.

Corollary 6.3. Let $k, r \geq 1, \tau$ a vocabulary, and $\mathrm{C}$ a class of $\tau$-structures of bounded local tree-width. Then there is an algorithm that, given a structure $\mathcal{A} \in \mathrm{C}$, computes an $(r, 2 k r)$-neighborhood cover $\mathcal{N}$ of $\mathcal{A}$ of size $\|\mathcal{N}\|=O\left(|A|^{1+(1 / k)}\right)$ in time $O\left(|A|^{1+(1 / k)}\right)$.

Proof: Since neighborhoods of radius $2 k r$ in structures in $\mathrm{C}$ have bounded tree-width, by Lemma 4.1 there is a constant $c$ such that for every structure $\mathcal{A} \in \mathrm{C}$, every $(r, 2 k r)$-neighborhood cover $\mathcal{N}$ of $\mathcal{A}$, and every $N \in \mathcal{N}$ we have

$$
\left\|\langle N\rangle^{\mathcal{A}}\right\| \leq c|N|
$$

This implies $\|\mathcal{A}\| \leq c\|\mathcal{N}\|$.

Our algorithm first computes the Gaifman graph $\mathcal{G}_{\mathcal{A}}$ of the input structure $\mathcal{A}$, which is possible in time $O(\|\mathcal{A}\|)$. Then it computes an an $(r, 2 k r)$-neighborhood cover $\mathcal{N}$ of $\mathcal{A}$ of size $\|\mathcal{N}\|=O\left(|A|^{1+(1 / k)}\right)$. By Lemma 6.2 and (1), this is possible in time $O(\|\mathcal{N}\|)=O\left(|A|^{1+(1 / k)}\right)$.

The following consequence of the proof of the previous corollary is worth being noted:

Corollary 6.4. Let $\tau$ be a vocabulary and $\mathrm{C}$ be a class of $\tau$-structures of bounded local tree-width. Then for every $k \geq 1$ there is a constant $c$ such that for all structures $\mathcal{A} \in \mathrm{C}$ we have $\|\mathcal{A}\| \leq c|A|^{1+(1 / k)}$.

As a matter of fact, a neighborhood cover is more than we need. Often, the following weaker notion of a tree cover leads to better results.

Definition 6.5. Let $r, w \geq 0$. An $(r, w)$-tree cover of a structure $\mathcal{A}$ is a family $\mathcal{T}$ of subsets of $A$ with the following properties:

(1) For every $a \in A$ there exists a $T \in \mathcal{T}$ such that $N_{r}^{\mathcal{A}}(a) \subseteq T$.

(2) For every $T \in \mathcal{T}$ we have $\operatorname{tw}\left(\langle T\rangle^{\mathcal{A}}\right) \leq w$.

Note that an $(r, s)$-neighborhood cover of a structure $\mathcal{A}$ is an $\left(r, \operatorname{ltw}^{\mathcal{A}}(s)\right)$-tree cover of $\mathcal{A}$. The following lemma is implicit in [12]:

Lemma 6.6 (Eppstein [12]). Let $r \geq 0$ and $\mathrm{C}$ be a class of graphs that is closed under taking minors and has bounded local tree-width. Let $f: \mathbb{N} \rightarrow \mathbb{N}$ be a function bounding the local tree-width of the graphs in C.

Then there is an algorithm that, given a graph $\mathcal{G} \in \mathrm{C}$, computes an $(r, f(2 r+1))$-tree cover $\mathcal{T}$ of $\mathcal{G}$ of size $\|\mathcal{T}\|=O(|G|)$ in time $O(|G|)$.

Proof: Let $\mathcal{G} \in \mathrm{C}$ and choose an arbitrary vertex $a_{0} \in G$. For $0 \leq i \leq j$, let $G[i, j]:=\{a \in G \mid i \leq$ $\left.d^{G}\left(a_{0}, a\right) \leq j\right\}$.

We claim that $\operatorname{tw}(\langle G[i, j]\rangle) \leq f(j-i+1)$. This is immediate if $i=0$ or $i=1$, because then $G[i, j] \subseteq N_{j}^{\mathcal{G}}\left(a_{0}\right)$. If $i>1$, we simply contract the connected subgraph $\langle G[0, i-1]\rangle^{\mathcal{G}}$ to a single vertex $b_{0}$. We obtain a minor $\mathcal{G}^{\prime}$ of $\mathcal{G}$, which is also an element of $\mathrm{C}$ by our assumption that $\mathrm{C}$ is closed under taking minors. $\mathcal{G}^{\prime}$ still contains the set $G[i, j]$ as it is, but this set is contained in $N_{j-i+1}^{\mathcal{G}^{\prime}}\left(b_{0}\right)$. This proves the claim. 
The claim implies that for all $r \geq 0$, the family $\mathcal{T}:=\{G[i, i+2 r] \mid i \geq 0\}$ is an $(r, f(2 r+1))$-tree cover of $\mathcal{G}$ of size at most $(2 r+1)|G|$. On input $\mathcal{G}$, we can choose an arbitrary $a_{0}$ and then compute this tree cover in linear time by breadth-first search.

The existence of a tree-cover of size linear in the size of the structure and a linear time algorithm computing such a cover is exactly what we need in our algorithms of the next section. This justifies the following definition:

Definition 6.7. A class $C$ of graphs is locally tree-decomposable if there is a function $g: \mathbb{N} \rightarrow \mathbb{N}$ and an algorithm that, given a structure $\mathcal{A} \in \mathrm{C}$ and an $r \in \mathbb{N}$, computes an $(r, g(r))$-tree cover of $\mathcal{A}$ of size $O(|A|)$ in time $O(|A|)$.

Examples 6.8. All examples of classes of structures of bounded local tree-width that we gave in Section 5 are actually locally tree-decomposable.

For Example 5.3, classes of structures of bounded valence, this is trivial: If $\mathcal{A}$ is a structure of valence $l$ and $r \geq 0$, then the family $\left\{N_{r}^{\mathcal{A}}(a) \mid a \in A\right\}$ is an $\left(r, l(l-1)^{r-1}\right)$-tree cover of $\mathcal{A}$.

For all other examples, it follows from Lemma .6.

The following proposition is an immediate consequence of the definition of locally tree-decomposable classes of structures:

Proposition 6.9. Let $\tau$ be a vocabulary and $\mathrm{C}$ be a locally tree-decomposable class of $\tau$-structures. Then there is a constant $c$ such that for all structures $\mathcal{A} \in \mathrm{C}$ we have $\|\mathcal{A}\| \leq c|A|$.

We close this section with an example showing that the analogue of Proposition 6.9 for classes of bounded local tree-width is wrong. Remember Corollary 6.4, though.

Example 6.10. We construct a class $\mathrm{C}$ of graphs of bounded local tree-width such that for every constant $c$ there is a graph $\mathcal{G} \in \mathrm{C}$ with $\|\mathcal{G}\| \geq c|G|$.

We use the following theorem due to Erdös [14]: For all $g, k \geq 1$ there exists a graph of girth greater than $g$ and chromatic number greater than $k$. Remember that the girth $g(\mathcal{G})$ of a graph $\mathcal{G}$ is the length of the shortest cycle in $\mathcal{G}$ and the chromatic number $\chi(\mathcal{G})$ of $\mathcal{G}$ is the least number of colors needed to color the vertices of $\mathcal{G}$ in such a way that no two adjacent vertices have the same color. It is easy to see that every graph $\mathcal{G}$ with $\chi(\mathcal{G}) \geq k$ has a connected subgraph $\mathcal{H}$ with average degree

$$
\frac{2\left|E^{\mathcal{H}}\right|}{\mathcal{V}^{\mathcal{H}}} \geq k-1
$$

(cf. 阿, p. 98).

The diameter of a connected graph $\mathcal{G}$ is the number $\operatorname{diam}(\mathcal{G}):=\max \left\{d^{\mathcal{G}}(a, b) \mid a, b \in G\right\}$.

We inductively construct a family $\left(\mathcal{G}_{i}\right)_{i \geq 1}$ of graphs as follows: $\mathcal{G}_{1}$ is the graph consisting of two vertices and an edge between them. Suppose now that $\mathcal{G}_{i}$ is already defined. Let $\mathcal{G}_{i+1}^{\prime}$ be a graph with $g\left(\mathcal{G}_{i+1}^{\prime}\right) \geq 2 \operatorname{diam}\left(\mathcal{G}_{i}\right)+1$ and $\chi\left(\mathcal{G}_{i+1}^{\prime}\right) \geq 2 i+3$. Let $\mathcal{G}_{i+1}$ be a connected subgraph of $\mathcal{G}_{i+1}^{\prime}$ with

$$
\frac{2\left|E^{\mathcal{G}_{i+1}}\right|}{\mathcal{V}^{\mathcal{G}_{i+1}}} \geq 2 i+2 .
$$

Clearly, $g\left(\mathcal{G}_{i+1}\right) \geq g\left(\mathcal{G}_{i+1}^{\prime}\right) \geq 2 \operatorname{diam}\left(\mathcal{G}_{i}\right)+1$.

Observe that for every $r \geq 1$ and every graph $\mathcal{G}$, if $2 r+1<g(\mathcal{G})$ then ${ }^{1 t w}{ }^{\mathcal{G}}(r) \leq 1$. Moreover, if $\mathcal{G}$ is connected then $\operatorname{ltw}^{\mathcal{G}}(r)=\operatorname{tw}(\mathcal{G})$ for all $r \geq \operatorname{diam}(\mathcal{G})$. For every $i \geq 1$ and $\operatorname{diam}\left(\mathcal{G}_{i}\right) \leq r<\operatorname{diam}\left(\mathcal{G}_{i+1}\right)$, we let $f(r):=\max \left\{\operatorname{tw}\left(\mathcal{G}_{i}\right), \operatorname{ltw}^{\mathcal{G}_{i+1}}(r)\right\}$. We claim that $\operatorname{ltw}^{\mathcal{G}_{i}}(r) \leq f(r)$ for all $i, r \geq 1$. This is obvious for $i=1$. For $i \geq 2$, we have to distinguish between three cases: If $r<\operatorname{diam}\left(\mathcal{G}_{i-1}\right) \leq \frac{1}{2}\left(g\left(\mathcal{G}_{i}\right)-1\right)$, then $\operatorname{ltw}^{\mathcal{G}_{i}}(r) \leq 1 \leq f(r)$. If $\operatorname{diam}\left(\mathcal{G}_{i-1}\right) \leq r<\operatorname{diam}\left(\mathcal{G}_{i}\right)$, then $\operatorname{ltw}^{\mathcal{G}_{i}}(r) \leq f(r)$ immediately by the definition of $f$. If $r \geq \operatorname{diam}\left(\mathcal{G}_{i}\right)$, then $\operatorname{ltw}^{\mathcal{G}_{i}}(r)=\operatorname{tw}\left(\mathcal{G}_{i}\right) \geq f(r)$.

Thus the class $\mathrm{C}:=\left\{\mathcal{G}_{i} \mid i \geq 1\right\}$ has bounded local tree-width. On the other hand, for every $i \geq 2$ we have $\left\|\mathcal{G}_{i}\right\| \geq\left|E^{\mathcal{G}_{i}}\right| \geq i\left|G_{i}\right|$.

\footnotetext{
${ }^{1}$ The hidden constants in the $O(\cdot)$-notation may depend on $r$.
} 


\section{The main algorithm}

Throughout this section, we fix a vocabulary $\tau$. We shall first prove two lemmas.

Lemma 7.1. Let $\mathrm{C}$ be a class of $\tau$-structures of bounded local tree-width and $r, w \geq 1$. Then there is an algorithm that solves the following problem in time $O(\|\mathcal{T}\|)$ :

Input: $\quad$ Structure $\mathcal{A} \in \mathrm{C},(r, w)$-tree cover $\mathcal{T}$ of $\mathcal{A}$.

Problem: $\quad$ Compute $K_{T}:=\left\{a \in A \mid N_{r}^{\mathcal{A}}(a) \subseteq T\right\}$ for all $T \in \mathcal{T}$.

Proof: Observe that $\|\mathcal{A}\|=O(\|\mathcal{T}\|)$, because by Lemma 4.1, for all $T \in \mathcal{T}$ we have $\left\|\langle T\rangle^{\mathcal{A}}\right\|=O(|T|)$.

Without loss of generality we can assume that $\mathcal{A}$ is a graph; if not we first compute its Gaifman graph. This is possible in time $O(\|\mathcal{A}\|)$. Furthermore, we can assume that the universe $A$ of $\mathcal{A}$ is the set $\{1, \ldots, n\}$ (see the appendix of [15] for details).

Let $T \in \mathcal{T}$, we show how to compute $K_{T}$ in time $O(|T|)$. We suppose that $T$ is given as a list $a_{1}, \ldots, a_{m}$ of its elements. Our algorithm is displayed in Figure 2. $K_{T}$ is computed iteratively, during the computation the current state of the set is stored in an array $K$ of length $n$. Note that we do not initialize the array to 0 in the beginning (we do not have the time to do that). Instead, we maintain a second "control array" $\Gamma$ of length $m$. The $j$ th entry of $\Gamma$ is $a_{j}$, for $j=1$ to $m$. $\Gamma$ is initialized to these values in Line 1 . Then at every stage in the computation, the set of all elements represented by the array $K$ is

$$
S(K):=\{a \in A \mid K[a] \in\{1, \ldots, m\} \text { and } \Gamma[K[a]]=a\} .
$$

After Line 2 is executed, we have $S(K)=T$.

Now the main loop in Lines 3-13 iteratively removes those elements from $S(K)$ whose neighbors are not all contained in $S(K)$. Thus after the $i$ th iteration we have

$$
S(K)=\left\{a \in T \mid N_{i}^{\mathcal{A}}(a) \nsubseteq T\right\} .
$$

So once we enter Line 15, we have $S(K)=K_{T}$. Lines 15-17 retrieve this set from the array $K$.

Let us analyze the running time of the algorithm. Lines 1 and 2 require time $O(m)$. To test the condition of Lines 7-8 requires constant time for each $b$. To test the condition of Lines 6-8, we have to step through the list of vertices adjacent to $a_{j}$ until either we find a $b$ that does not satisfy the condition or we have checked all neighbors. This requires a constant amount of work for every edge with one endpoint $a_{j}$ and the other endpoint in $S(K)$ and an additional constant amount of work in case we find a neighbor not in $S(K)$. Thus the execution of the loop in lines 5-11 requires time $O\left(m+\left|E^{\mathcal{A}} \cap T^{2}\right|\right) \leq O\left(\left\|\langle T\rangle^{\mathcal{A}}\right\|\right)=O(m)$. The loop in Lines 12-14 also requires time $O(m)$. Thus every iteration of the main loop requires time $O(m)$. Since we treat the number $r$ of iterations as constant, the overall time required by Lines $3-15$ is $O(m)$. Since $K_{T} \subseteq T$, Lines $16-19$ also require time $O(m)$.

Lemma 7.2. Let $\mathrm{C}$ be a class of structures of bounded local tree-width and $r, m \geq 1$. Then the following problem can be solved in time $O(|A|)$ :

Input: $\quad$ Structure $\mathcal{A} \in \mathrm{C}$, set $P \subseteq A$.

Problem: Decide if there exist $a_{1}, \ldots, a_{m} \in P$ such that $d^{\mathcal{A}}\left(a_{i}, a_{j}\right)>r$.

Proof: Let $f: \mathbb{N} \rightarrow \mathbb{N}$ be a function bounding the local tree-width of the structures in C.

Let $\mathcal{A} \in \mathrm{C}$ and $P \subseteq A$. Our algorithm is displayed in Figure 3 . It proceeds in two phases.

In the first phase (Lines 1-12) it iteratively computes elements $a_{1}, \ldots, a_{i} \in P$, for some $i \leq m$, such that $d^{\mathcal{A}}\left(a_{i}, a_{j}\right)>r$ for $1 \leq i<j \leq l$ and either $l=m$ or for all $b \in P$ there is an $i \leq l$ such that $b \in N_{r}^{\mathcal{A}}\left(a_{i}\right)$. If $l=m$, the algorithm accepts. If $l=0$, i.e. $P=\emptyset$, then it rejects. Otherwise, it goes into the second phase (Lines 13-18). 


$$
\begin{aligned}
& \text { Input: } \mathcal{A}, T=\left\{a_{1}, \ldots, a_{m}\right\} \subseteq A \\
& \text { 1. for } j=1 \text { to } m \text { do } \Gamma[j]:=a_{j} \text { od } \\
& \text { 2. for } j=1 \text { to } m \text { do } K\left[a_{j}\right]:=j \text { od } \\
& \text { 3. for } i=1 \text { to } r \text { do } \\
& \text { 4. temp }:=\emptyset \\
& \text { 5. for } j=1 \text { to } m \text { do } \\
& \text { 6. if } a_{j} \text { has a neighbor } b \text { such that } \\
& \text { 7. } \quad(K[b] \notin\{1, \ldots, m\} \\
& \text { 8. } \quad \text { or }(K[b] \in\{1, \ldots, m\} \text { and } \Gamma[K[b]] \neq b)) \text { then } \\
& \text { 9. } \quad \text { temp }:=\operatorname{temp} \cup\left\{a_{j}\right\} \\
& \text { 10. fi } \\
& \text { 11. od } \\
& \text { 12. for all } a \in \text { temp do } \\
& \text { 13. } K[a]:=0 \\
& 14 . \quad \text { od } \\
& \text { 15. od } \\
& \text { 16. } K_{T}:=\emptyset \\
& \text { 17. for } j=1 \text { to } m \text { do } \\
& \text { 18. if } K\left[a_{j}\right]=j \text { then } K_{T}:=K_{T} \cup\left\{a_{j}\right\} \text { fi } \\
& \text { 19. od }
\end{aligned}
$$

Output: $K_{T}$

Figure 2 .

When the algorithm enters Line 13, we have $P \subseteq N_{r}^{\mathcal{A}}\left(\left\{a_{1}, \ldots, a_{l}\right\}\right)$. Let $\mathcal{H}:=\left\langle N_{2 r}^{\mathcal{A}}\left(\left\{a_{1}, \ldots, a_{l}\right\}\right)\right.$. Then for all $b, b^{\prime} \in P$ we have $d^{\mathcal{A}}\left(b, b^{\prime}\right) \leq r \Longleftrightarrow d^{\mathcal{H}}\left(b, b^{\prime}\right) \leq r$, because $P \subseteq N_{r}^{\mathcal{A}}\left(\left\{a_{1}, \ldots, a_{l}\right\}\right)$ and thus every path of length at most $r$ between two elements of $P$ must be contained in $H$. Thus there exist $b_{1}, \ldots, b_{m} \in P$ such that $d^{\mathcal{A}}\left(b_{i}, b_{j}\right)>r$ if, and only if, there exist $b_{1}, \ldots, b_{m} \in P$ such that $d^{\mathcal{H}}\left(b_{i}, b_{j}\right)>r$, i.e. if the condition in Line 14 is satisfied. Thus the algorithm is correct

To estimate the running time, we note that $\left\|\left\langle N_{r}^{\mathcal{A}}\left(a_{i}\right)\right\rangle^{\mathcal{A}}\right\|=O\left(\left|N_{r}^{\mathcal{A}}\left(a_{i}\right)\right|\right)$, because $\mathrm{C}$ is a class of bounded local tree-width. Since we treat $r$ and $m$ as constants, Lines 1-13 require time $O(|A|)$. It is easy to see that $\operatorname{tw}(\mathcal{H}) \leq \operatorname{ltw}^{\mathcal{A}}(2 l r) \leq f(2 l r)$. Thus the condition in Line 14 can also be checked in time $O(|A|)$ by Courcelle's Theorem 4.2 .

We are now ready to prove our main results, Theorems 1.1 and 1.2. Recall the statements:

Let $\mathrm{C}$ be a class of structures of bounded local tree-width and $\varphi$ a sentence of first-order logic.

(1) For every $k \geq 1$ there is an algorithm that decides whether a given structure $\mathcal{A} \in \mathrm{C}$ satisfies $\varphi$ in time $O\left(|A|^{1+(1 / k)}\right)$.

(2) If $\mathrm{C}$ is locally tree-decomposable, then there is an algorithm that solves the problem in time $O(|A|)$. 


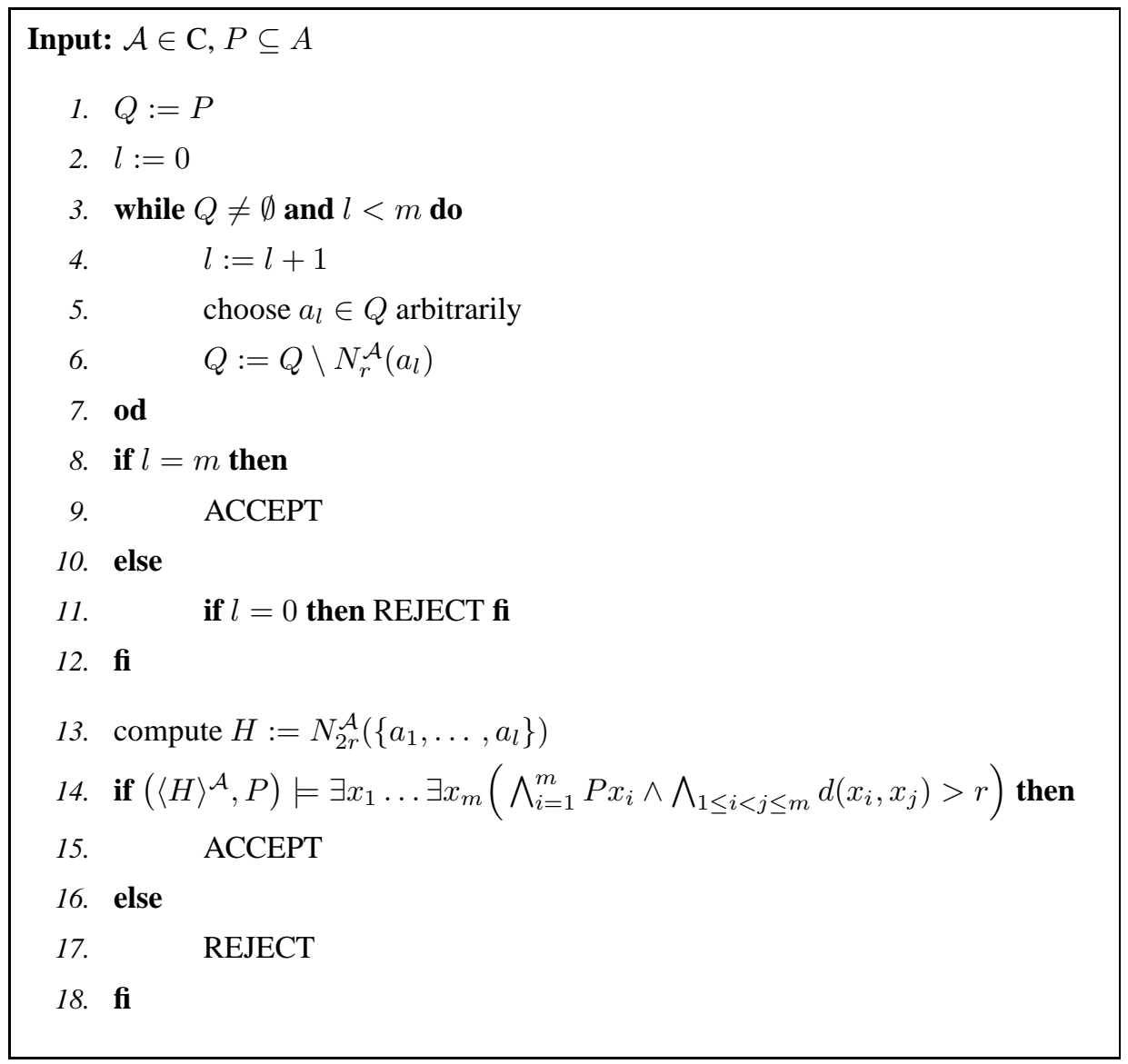

Figure 3.

Proof: We describe the algorithm for (1) and then explain how it has to be modified to obtain (2).

By Gaifman's Theorem 3.1, without loss of generality we can assume that $\varphi$ is of the form

$$
\exists x_{1} \ldots \exists x_{m}\left(\bigwedge_{1 \leq i<j \leq m} d\left(x_{i}, x_{j}\right)>2 r \wedge \bigwedge_{1 \leq i \leq m} \psi\left(x_{i}\right)\right),
$$

for suitably chosen $r, m \geq 1$ and an $r$-local $\psi$.

Let $k \geq 1$ and $f: \mathbb{N} \rightarrow \mathbb{N}$ be a function bounding the local tree-width of the structures in C. Let $\tau$ be the vocabulary of the sentence $\varphi$; without loss of generality we can assume that all structures in $\mathrm{C}$ are $\tau$-structures.

Figure 4 shows our algorithm.

To see that the algorithm is correct, note that since $\psi(x)$ is $r$-local we have $P=\{a \in A \mid \mathcal{A} \models \psi(a)\}$.

So we shall prove that the algorithm can be implemented as an $O\left(n^{1+(1 / k)}\right)$-algorithm, where $n:=|A|$ is the cardinality of the input structure.

Line 1 requires time $O\left(n^{1+(1 / k)}\right)$ by Corollary 6.3. Lines $2-4$ require time $O(\|\mathcal{N}\|)$ by Lemma 7.1 . For every $N \in \mathcal{N}$, Line 6 requires time $O(|N|)$ by Lemma 4.3. Thus the loop in Lines 5-7 also requires time $O(\|\mathcal{N}\|)$. Clearly, Line 8 can be performed in time $O(\|\mathcal{N}\|)$, and the condition in Line 9 can be checked in time $O(|A|)$ by Lemma 7.2. Thus the overall running time is $O(\|\mathcal{N}\|)=O\left(n^{1+(1 / k)}\right)$.

It remains to prove (2), but this is very easy now. Instead of a neighborhood cover, in Line 1 of the algorithm we compute tree cover of linear size. This can be done in linear time by the definition of a locally 


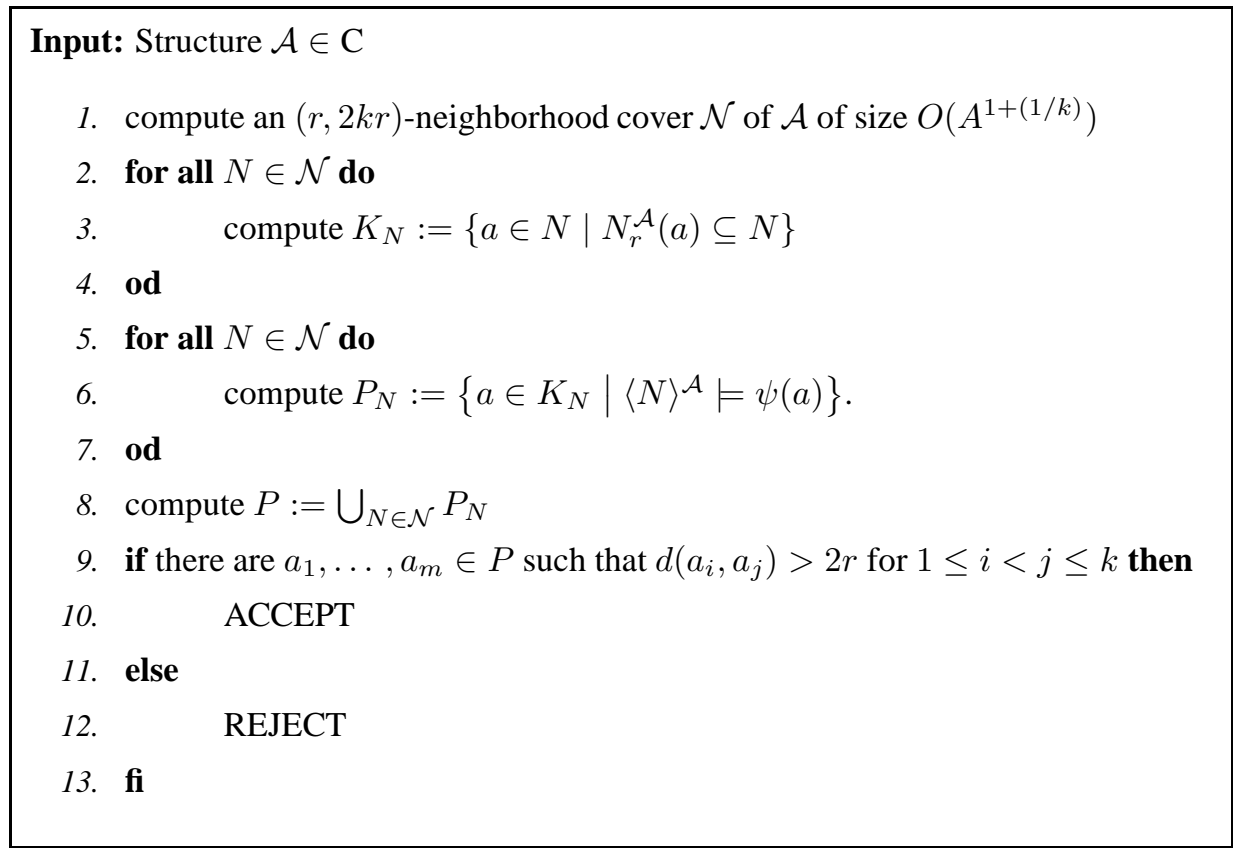

Figure 4.

tree-decomposable class of graphs. Since the running time of the rest of the algorithm is linear in the size of the cover, we obtain a linear time algorithm.

\section{Concluding remarks}

Uniformity. A close look at our proofs shows that actually for each locally tree-decomposable class C of structures there is a recursive function $f: \mathbb{N} \rightarrow \mathbb{N}$ and an algorithm that decides, given a first-order sentence $\varphi$ and a structure $\mathcal{A} \in \mathrm{C}$, whether $\mathcal{A} \models \varphi$ in time $O(f(\|\varphi\|)|A|)$, where $\|\varphi\|$ denotes the length of the sentence $\varphi$. We can obtain an anlogous uniform version of Theorem 1.2 .

We stated and proved non-uniform versions of the theorems for the sake of a clearer presentation.

Dependence on the formula size. Our algorithm heavily depends on the size of the formula $\varphi$, roughly the hidden multiplicative constant is $k$-fold exponential in the length of $\varphi$, where $k$ is the number of quantifieralternations in $\varphi$.

Practical Considerations. The large hidden constants seem to make our algorithms useless for practical purposes. Nevertheless, let us briefly discuss a few more practical aspects.

The main factor contributing to the large constants is the complexity of the formulas, in particular the number of quantifier alternations. However, if we think of a database application, we will usually only have to handle very simple formulas. As matter of fact, most database queries are so called conjunctive queries; they can be defined by first-order formulas of the form $\exists x_{1} \ldots \exists x_{k}\left(\alpha_{1} \wedge \ldots \wedge \alpha_{m}\right)$. Such formulas do not have any quantifier alternation. Moreover, when handling conjunctive queries we can avoid the second very costly part hidden in our algorithms, namely the transformation of a formula according to Gaifman's theorem. For all we know, such a transformation may blow up the formula size by a nonelementary factor. For conjunctive queries, we can avoid Gaifman's theorem and instead use algorithmic techniques similar to those in [13]. With these techniques, the dependence on the formula size can be reduced to a singly exponential factor, which seems acceptable because usually in practice we have to 
evaluate small formulas (queries) in large structures (databases). The third costly factor is to compute treedecompositions. Bodlaender's linear time algorithm is only of theoretical interest due to very large hidden constants. More promising seems to be an algorithm due to Reed [22] (improving an earlier algorithm due to Robertson and Seymour [24]). For an input graph of tree-width $w$ and size $n$, this algorithm only computes a tree-decomposition of width at most $4 w$. Its running time is not linear in $n$ (as Bodlaenders), but $O(n \log (n))$. However, the algorithm is simple and the hidden constants, though exponentially depending on $w$, do not seem too large for small values of $w$. Let us also remark that there are much more efficient algorithms for computing small width tree-decompositions of planar graphs of small radius [12].

Nevertheless, as they stand our results are mostly theoretical. Similarly to Courcelle's Theorem [8], their main benefit is to provide a quick and simple way to recognize a property as being linear time computable on certain classes of graphs. Analyzing the combinatorics of the specific property then, one may also find a practical algorithm.

Further Research. Although Example 6.10 shows that for classes $\mathrm{C}$ of bounded local tree-width we cannot expect an algorithm deciding a first-order property of structures $\mathcal{A} \in \mathrm{C}$ in time $O(|A|)$, it does not rule out an $O(\|\mathcal{A}\|)$-algorithm. To obtain such an algorithm, it would be sufficient to find, for every $r \geq 1$, a $w \geq 1$ and an $O(\|\mathcal{A}\|)$-algorithm that computes an $(r, w)$-tree cover of a structure $\mathcal{A} \in \mathrm{C}$.

As we mentioned, one of the main factors contributing to the heavy dependence of the running time of our algorithms on the size of the formula is the transformation into a "local formula" according to Gaifman's theorem. Though this transformation is clearly effective, as far as we know its complexity has not been studied. We do expect this complexity to be non-elementary, but this does not rule out the existence of more efficient algorithms for particular classes of formulas (such as existential formulas) or the existence of good heuristics.

In general, we consider it as one of the main challenges for further research to reduce the dependence on the formula size (not only in our results, but also in Courcelle's theorem). For example, is there an algorithm that decides, given a first-order sentence $\varphi$ and a planar graph $\mathcal{G}$ (or a tree, or just a word), whether $\mathcal{G} \models \varphi$ in time $O\left(2^{|\varphi|} n^{c}\right)$ for some fixed-constant $c$ ?

\section{References}

[1] A.V. Aho, J.E. Hopcroft, and J.D. Ullman. The Design and Analysis of Computer Algorithms. Addison-Wesley, 1974.

[2] A.V. Aho and J.D. Ullman. The universality of data retrieval languages. In Conference Record of the Sixth Annual ACM Symposium on Principles of Programming Languages, pages 110-120, 1979.

[3] B. Awerbuch, B. Berger, L. Cowen, and D. Peleg. Near-linear cost sequential and distributed constructions of sparse neighborhood covers. In Proceedings of the 34th Annual IEEE Symposium on Foundations of Computer Science, pages 638-647, 1993.

[4] B. Awerbuch and D. Peleg. Sparse partitions. In Proceedings of the 31 st Annual IEEE Symposium on Foundations of Computer Science, pages 503-513, 1990.

[5] B.S. Baker. Approximation algorithms for NP-complete problems on planar graphs. Journal of the ACM, 41:153-180, 1994.

[6] H.L. Bodlaender. A linear-time algorithm for finding tree-decompositions of small treewidth. SIAM Journal on Computing, 25:1305-1317, 1996.

[7] H.L. Bodlaender. Treewidth: Algorithmic techniques and results. In I. Privara and P. Ruzicka, editors, Proceedings 22nd International Symposium on Mathematical Foundations of Computer Science, MFCS'97, volume 1295 of Lecture Notes in Computer Science, pages 29-36. Springer-Verlag, 1997.

[8] B. Courcelle. Graph rewriting: An algebraic and logic approach. In J. van Leeuwen, editor, Handbook of Theoretical Computer Science, volume 2, pages 194-242. Elsevier Science Publishers, 1990. 
[9] R. Diestel. Graph Theory. Springer-Verlag, second edition, 2000.

[10] R.G. Downey and M.R. Fellows. Parameterized Complexity. Springer-Verlag, 1999.

[11] R.G. Downey, M.R. Fellows, and U. Taylor. The parameterized complexity of relational database queries and an improved characterization of $W[1]$. In Bridges, Calude, Gibbons, Reeves, and Witten, editors, Combinatorics, Complexity, and Logic - Proceedings of DMTCS '96, pages 194-213. Springer-Verlag, 1996.

[12] D. Eppstein. Diameter and treewidth in minor-closed graph families. Algorithmica. To appear.

[13] D. Eppstein. Subgraph isomorphism in planar graphs and related problems. Journal of Graph Algorithms and Applications, 3:1-27, 1999.

[14] P. Erdös. Graph theory and probability. Canadian Journal of Mathematics, 11:34-38, 1959.

[15] J. Flum, M. Frick, and M. Grohe. Query-evaluation via tree-decompositions. Available at http://sunpool.mathematik.uni-freiburg.de/home/grohe/pub.html, 2000. Submitted for publication.

[16] J. Flum and M. Grohe. Fixed-parameter tractability and logic. Available at http://xxx.lanl.gov/abs/cs.CC/9910001, 1999. Submitted for publication.

[17] H. Gaifman. On local and non-local properties. In Proceedings of the Herbrand Symposium, Logic Colloquium '81. North Holland, 1982.

[18] M.R. Garey, D.S. Johnson, and L. Stockmeyer. Some simplified NP-complete graph problems. Theoretical Computer Science, 1:237-267, 1976.

[19] N. Immerman. Upper and lower bounds for first-order expressibility. Journal of Computer and System Sciences, 25:76-98, 1982.

[20] C.H. Papadimitriou and M. Yannakakis. On the complexity of database queries. In Proceedings of the 16th ACM Symposium on Principles of Database Systems, pages 12-19, 1997.

[21] D. Peleg. Distance-dependent distributed directories. Information and Computation, 103:270-298, 1993.

[22] B. Reed. Finding approximate separators an computing tree-width quickly. In Proceedings of the 24th ACM Symposium on Theory of Computing, pages 221-228, 1992.

[23] N. Robertson and P.D. Seymour. Graph minors III. Planar tree-width. Journal of Combinatorial Theory, Series B, 36:49-64, 1984.

[24] N. Robertson and P.D. Seymour. Graph minors XIII. The disjoint paths problem. Journal of Combinatorial Theory, Series B, 63:65-110, 1995.

[25] D. Seese. Linear time computable problems and first-order descriptions. Mathematical Structures in Computer Science, 6:505-526, 1996.

[26] P. van Emde Boas. Machine models and simulations. In J. van Leeuwen, editor, Handbook of Theoretical Computer Science, volume 1, pages 1-66. Elsevier Science Publishers, 1990.

[27] M. Y. Vardi. The complexity of relational query languages. In Proceedings of the 14th ACM Symposium on Theory of Computing, pages 137-146, 1982.

[28] M. Yannakakis. Perspectives on database theory. In Proceedings of the 36th Annual IEEE Symposium on Foundations of Computer Science, pages 224-246, 1995. 